\title{
LHomme
}

L'HOMME Revue française d'anthropologie

239-240 | 2021

Expériences initiatiques du genre

\section{Jean Tsaboto, Mutations et révolutions au XIX siècle chez les Antemoro de Madagascar}

\section{Sophie Goedefroit}

\section{OpenEdition}

\section{Journals}

Édition électronique

URL : https://journals.openedition.org/lhomme/41354

DOI : 10.4000//homme.41354

ISSN : 1953-8103

\section{Éditeur}

Éditions de l'EHESS

\section{Édition imprimée}

Date de publication : 1 décembre 2021

Pagination : $340-342$

ISBN : 978-2-7132-2868-1

ISSN : 0439-4216

Référence électronique

Sophie Goedefroit, « Jean Tsaboto, Mutations et révolutions au xixe siècle chez les Antemoro de Madagascar », L'Homme [En ligne], 239-240 | 2021, mis en ligne le 01 décembre 2021, consulté le 15 décembre 2021. URL : http://journals.openedition.org//homme/41354 ; DOI : https://doi.org/10.4000/ Ihomme.41354

Ce document a été généré automatiquement le 15 décembre 2021.

(c) École des hautes études en sciences sociales 


\title{
Jean Tsaboto, Mutations et révolutions au XIX ${ }^{e}$ siècle chez les Antemoro de Madagascar
}

\author{
Sophie Goedefroit
}

\section{RÉFÉRENCE}

Jean Tsaboto, Mutations et révolutions au XIXe siècle chez les Antemoro de Madagascar. Éd. par Philippe Beaujard. Paris, Hémisphères-Maisonneuve \& Larose, 2021, 660 p., bibl., index, gloss., ill., fig., tabl., cartes.

1 Jean Tsaboto, auteur de cet ouvrage, est décédé en 2003 après avoir effectué un long et minutieux terrain de recherche chez les Antemoro du Sud-Est de Madagascar, dont il était issu et auxquels il consacra une thèse de doctorat en histoire soutenue la même année. C'est à l'anthropologue et historien spécialiste de Madagascar Philippe Beaujard, qui a légèrement retravaillé le manuscrit et l'a annoté, que nous devons cette édition posthume.

2 Cet imposant volume est remarquable par l'importance de la diversité des sources mobilisées et par la précision et la rigueur de leur traitement. Les sorabe, manuscrits arabico-malgaches consignant les faits historiques du pays antemoro depuis le $\mathrm{XVII}^{\mathrm{e}}$ siècle, $\mathrm{y}$ sont confrontés aux récits des voyageurs et aux archives précoloniales, notamment aux échanges de courriers entre les souverains merina qui se sont succédé sur le trône de Madagascar et leurs gouverneurs de province. Les rapports des missionnaires des différentes congrégations religieuses qui s'installèrent en pays antemoro dès le XIX $x^{e}$ siècle sont également examinés. Jean Tsaboto compare donc les faits tels qu'ils sont relatés par les Antemoro eux-mêmes dans leurs manuscrits avec les témoignages émanant de regards extérieurs à cette société. Il fait en cela œuvre d'historien. Mais il va encore plus loin en menant parallèlement une recherche ethnographique de terrain, afin de collecter les mythes et traditions orales auprès des 
différents groupes constitutifs de cette société. Il consulte les lieux historiques comme il le fait des archives, reconstitue les généalogies, explore les connaissances magicoreligieuses et astrologiques, dissèque de nombreuses variantes de rituels majeurs. Il n'omet pas non plus de réfléchir à la qualité de certaines des informations qui lui ont été livrées, et ce, en raison de sa propre position au sein de la société antemoro: son statut d'Antemoro descendant de roturiers a en effet pu être un obstacle pour accéder aux témoignages des aristocrates. Ce travail de terrain complété par une analyse réflexive ajoute donc à cette recherche érudite d'historien une dimension ethnologique. Il convient de souligner l'exemplarité de cette démarche qui dépasse largement son objet: les transformations sociales, les repositionnements de pouvoir et les mutations qui se déroulent au fil de l'histoire de Madagascar au sein d'une société très particulière.

Les Antemoro occupent une place importante dans l'historiographie de Madagascar et ce, à plusieurs titres. En premier lieu, nous disposons de témoignages écrits remontant $\mathrm{au} \mathrm{Xv} \mathrm{e}^{\mathrm{e}}$ siècle relatant l'arrivée de groupes islamisés sur la côte Sud-Est qui, après avoir chassé les anciens groupes d'origine austronésienne, furent à l'origine du royaume antemoro. En second lieu, ces groupes islamisés sont reconnus pour avoir introduit à Madagascar une écriture en caractères arabes, qui servira à l'inscription d'un savoir magico-religieux arabo-persan, mais aussi à la conservation des chroniques du royaume antemoro (les sorabe). Elle sera ensuite utilisée comme écriture "officielle " par les souverains de Madagascar à la cour de l'Imerina, avant que l'alphabet latin ne la supplante. Tout en apparaissant très tôt dans la littérature ancienne, les Antemoro ont également eu une influence indéniable dans la diffusion d'un savoir ésotérique islamisé (l'astrologie solaire notamment) dans toute l'île, ainsi que dans l'idéologie et la constitution des appareils politiques de nombreux royaumes malgaches au cours des $\mathrm{XVI}^{\mathrm{e}}$ et XVII ${ }^{\mathrm{e}}$ siècles.

4 Jean Tsaboto a entrepris de retracer l'histoire sociale et politique des Antemoro depuis la fondation de leur royaume jusqu'à son déclin au $\mathrm{xIX}^{\mathrm{e}}$ siècle. Au fil de son ouvrage divisé en trois parties chronologiques, il décortique l'équilibre interne d'une organisation dualiste opposant les aristocrates, ou «pseudo-castes » (sic) se partageant le pouvoir, aux autres strates de la société : roturiers, esclaves et parias. Il nous dépeint le tableau d'une société fortement hiérarchisée qui, au gré du temps et des interventions extérieures, va connaître de profonds bouleversements. À partir du $\mathrm{XIX}^{\mathrm{e}}$ siècle, en effet, les rivalités inter-claniques fissurèrent doucement l'appareil monarchique, que le lien de suzeraineté à la royauté de l'Imerina affaiblit encore. Les corvées, les impôts, l'enrôlement dans l'armée merina de jeunes Antemoro de toutes origines sociales brouillèrent le système de classes. Surtout, l'implantation des écoles et l'alphabétisation donnèrent à tous l'accès à l'écriture et à une certaine promotion sociale, alors que, jusque-là, seule une portion de l'élite aristocratique était détentrice des sorabe, de l'écriture et du savoir par lesquels leur pouvoir trouvait sa légitimité. La destruction des «idoles et des charmes » ordonnée par la reine merina Ranavalona II en 1869, l'arrivée de prédicateurs, la construction de temples et d'églises, les conversions forcées au christianisme contribuèrent également à la remise en cause des privilèges détenus par certaines classes sociales. De très belles pages sont consacrées à la contestation des privilèges de l'endogamie et de l'égorgement des animaux, qui provoquèrent conflits, révoltes et « révolution» (sic) au cours de la seconde moitié du $\mathrm{XIX}^{\mathrm{e}}$ siècle en pays antemoro. 
5 Jean Tsaboto ne s'enferme pas pour autant dans une monographie. Il décrit de façon précise et parfois inédite la diversité des rapports ou des liens que les Antemoro ont pu entretenir avec les différents groupes sociaux de Madagascar, tels que les Betsileo, les Bara, les Tanala, les Bestimisaraka, les Antesaka, les Antambaoka et, bien sûr, les Merina. Il confirme ainsi leur influence sur les autres royaumes ou groupes sociaux de Madagascar, mais surtout, la détaille, l'interroge et nous fait nous interroger en retour. C'est là un des apports notables de cet ouvrage pour les non-spécialistes. En effet, on ne peut qu'adhérer, par exemple, à l'hypothèse posée dès le début du livre selon laquelle l'idéologie patrilinéaire, portée par les islamisés fondateurs du royaume Antemoro, a fortement infléchi le principe de filiation cognatique d'origine austronésienne en vigueur dans la structure lignagère prédynastique et s'est propagée, couplée à l'idéologie d'un pouvoir centralisé, dans l'ensemble de l'île à partir du Xvi ${ }^{\mathrm{e}}$ siècle. C'est une pensée répandue dans la littérature et rarement remise en question. Or, la démonstration est faite tout au long de l'ouvrage de l'importance cruciale, dans la succession au pouvoir et dans les rapports de groupes, de l'oncle maternel. De sorte que le droit de primogéniture et d'aînesse lignagère imposé comme un dogme dans l'élection au pouvoir est, par l'évidence des données fournies, battu en brèche. De même, les pratiques d'adoption, très peu décrites à Madagascar, sont mises en exergue. Cette présence de principes austronésiens dans les modes de filiation et d'organisation politique chez les Antemoro islamisés nous force à repenser la nature de leurs influences dans la constitution de certains royaumes et à réinterpréter leur histoire.

6 La lecture de cet ouvrage fournit un trésor d'informations pouvant inspirer tous ceux qui, anthropologues ou historiens, s'intéressent à Madagascar et aux mondes austronésiens. On ne peut que le recommander également pour son traitement exemplaire des données d'archives et de terrain. 